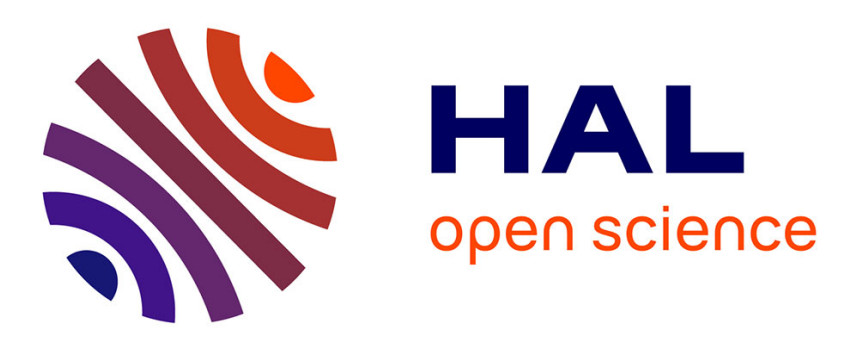

\title{
Prévisions des isolements acoustiques normalisés par une meilleure prise en compte des transmissions latérales. Approche expérimentale
}

M. Meisser

\section{- To cite this version:}

M. Meisser. Prévisions des isolements acoustiques normalisés par une meilleure prise en compte des transmissions latérales. Approche expérimentale. Journal de Physique IV Proceedings, 1994, 04 (C5), pp.C5-171-C5-174. 10.1051/jp4:1994532 . jpa-00253027

\section{HAL Id: jpa-00253027 https://hal.science/jpa-00253027}

Submitted on 1 Jan 1994

HAL is a multi-disciplinary open access archive for the deposit and dissemination of scientific research documents, whether they are published or not. The documents may come from teaching and research institutions in France or abroad, or from public or private research centers.
L'archive ouverte pluridisciplinaire HAL, est destinée au dépôt et à la diffusion de documents scientifiques de niveau recherche, publiés ou non, émanant des établissements d'enseignement et de recherche français ou étrangers, des laboratoires publics ou privés. 


\title{
Prévisions des isolements acoustiques normalisés par une meilleure prise en compte des transmissions latérales. Approche expérimentale
}

\author{
M. MEISSER
}

Laboratoire d'Application des Sciences Acoustiques, BP. 30, 78601 Maisons Laffitte cedex, France

\begin{abstract}
Résumé : Le LASA, avec son logiciel MOD.L, propose une nouvelle méthode permettant d'évaluer et d'optimiser les isolements acoustiques normalisés, en $d B(A)$, par une détermination de chaque transmission directe et latérale. Basée sur une approche expérimentale, cette méthode est compatible avec la théorie, développée notamment au niveau européen. Dans le cas des constructions traditionnelles, le logiciel MOD.L réduit l'incertitude liée à la prévision des résultats. Dans le cas des constructions avec des parois de séparation légères et des parois latérales filantes, il donne des évaluations très proches des résultats de mesures alors que la méthode classique utilisée habituellement présente de graves lacunes.
\end{abstract}

En France, dans le domaine de l'isolation acoustique, les constructeurs d'immeubles d'habitation neufs doivent satisfaire des obligations de résultats. Les concepteurs et les entrepreneurs qui ne sont pas des spécialistes en acoustique doivent donc pouvoir disposer d'outils simples leur permettant de prévoir les prestations à mettre en oeuvre en évaluant les isolements acoustiques correspondants.

La méthode d'évaluation des isolements acoustiques la plus utilisée actuellement consiste à calculer la part de transmission directe par la paroi de séparation et à évaluer forfaitairement l'ensemble des transmissions latérales.

$$
D n=[R+10 * \log (0.32 * V / S)]-a \quad[\mathrm{~dB}(\mathrm{~A})]
$$

où Dn est l'isolement acoustique normalisé pour une durée de réverbération de référence de 0.5 seconde

$\checkmark$ est le volume du local réception en $\mathrm{m}^{3}$

$S$ est la surface de la paroi de séparation commune aux deux locaux, en $\mathrm{m}^{2}$ a correspond à l'évaluation forfaitaire des transmissions latérales.

$a=5+S_{r} / 10-N$

où $S_{\xi}$ est la surface des parois légères et rigides liées à la paroi de séparation

$\mathrm{N}$ est le nombre parois latérales doublées de complexes en laine minérale avec une plaque de plâtre. 
Cette méthode donne des résultats satisfaisants lorsque les constructions sont traditionnelles avec des parois latérales rigides ou souples, non filantes, doublées ou non et des parois de séparation non doublées. Pour ces configurations, plus de $90 \%$ des résultats de mesures sont à $3 \mathrm{~dB}(\mathrm{~A})$ ou moins des prévisions. Encore faut-il préciser que ces écarts sont valables pour des dimensions de locaux émission et réception observées habituellement dans les logements.

Par contre, lorsque les parois latérales sont filantes, lorsque les parois latérales sont doublées de systèmes "masse-ressort" lorsque les parois de séparation sont légères de type "masse-ressort-masse" ou lorsque les dimensions des locaux sont très différentes de celles des pièces standard, les écarts "prévisions-mesures" peuvent être beaucoup plus importants avec la méthode ci-dessus.

Dans ces derniers cas, l'amélioration des prévisions ne peut être obtenue que par une évaluation plus précise de chacune des transmissions latérales.

Avant de continuer, il faut noter que les écarts entre les prévisions et les résultats de mesures sont dus à la combinaison de quatre sortes d'imprécisions :

- L'imprécision de la méthode de prévision utilisée

- L'imprécision liée aux matériaux et matériels utilisés

- L'imprécision liée à la mise en oeuvre

- L'imprécision de la méthode de mesures.

\section{La méthode proposée par le LASA}

\section{Buts de la méthode :}

- Evaluer la part de chaque paroi (paroi de séparation et quatre parois latérales) dans l'isolement acoustique normalisé entre deux locaux

- Mettre au point une méthode suffisamment simple pour être utilisable par les concepteurs et entrepreneurs non spécialistes des problèmes d'acoustique du bâtiment

- Permettre l'optimisation des solutions en vue d'obtenir un isolement acoustique normalisé déterminé.

Pour atteindre ces trois objectifs, nous avons utilisé dans un premier temps 150 cas pour lesquels nous disposions des plan, du descriptif des parois mises en place et des résultats de mesures.

\section{Hypothèses de départ :}

- La transmission directe par la paroi de séparation est évaluée de façon suffisamment précise par la méthode traditionnelle : $R+10^{*} \log 0.32 \mathrm{~V} / \mathrm{S}$.

- La transmission par une paroi latérale dépend des indices d'affaiblissement acoustique de la paroi latérale $R_{1}$ et de la paroi de séparation $R_{0}$.

- La transmission latérale dépend également du type de liaison entre la paroi latérale et la paroi de séparation

- Chaque paroi latérale se comporte comme si elle était affectée d'un indice d'affaiblissement acoustique fictif dépendant des deux indices ci-dessus et du type de liaison entre les parois.

$$
R_{1}^{\prime}=f\left(R_{0}, R_{1}\right)+C_{i}+g \text { (dimensions des locaux) }
$$


Des mesures faites il y a plus de 15 ans au C.E.B.T.P. dans une cellule permettant de tester les jonctions en forme de " $T$ " avaient montré que le chemin de transmission d'une paroi " $j$ " vers une paroi "j" a la même importance que le chemin de "j" vers "i". C'est pourquoi, nous avons très rapidement considéré que l'indice d'affaiblissement acoustique fictif correspondant à la paroi latérale considérée dépendait de la moyenne des indices d'affaiblissement acoustique des deux parois liées. En partant de cette constatation, nous avons déterminé des valeurs de facteurs " $\mathrm{C}_{1}$ " pour plusieurs configurations dont les résultats sont bien connus (trois parois latérales identiques à la paroi de séparation, même cas que le précédent avec une des parois remplacée par une paroi légère et rigide, même cas que le premier avec une des parois latérales remplacée par une paroi souple, et cas de trois parois lourdes identiques en parois latérales et d'une paroi de séparation de type "masse-ressort-masse").

Cela conduisait à des facteurs de liaison différents suivant que les jonctions se faisaient entre parois lourdes et rigides ou entre paroi lourde et paroi légères rigides. Ainsi les termes étaient différents et conduisaient à des écarts suivant le terme choisi pour évaluer le résultat à la frontière entre les parois lourdes et les parois légères. C'est pourquoi, nous avons refait une nouvelle approche en considérant que le facteur de jonction dépendait de la valeur absolue de " $R_{0}-R_{1}$ ", différence entre les indices d'affaiblissement des parois liées.

Pour tenir compte du fait que les jonctions peuvent être en forme de " $T$ " ou en forme de croix, et des doublages éventuels de type "masse-ressort", nous avons estimé des variations du facteur de liaison en examinant les deux schémas suivants.

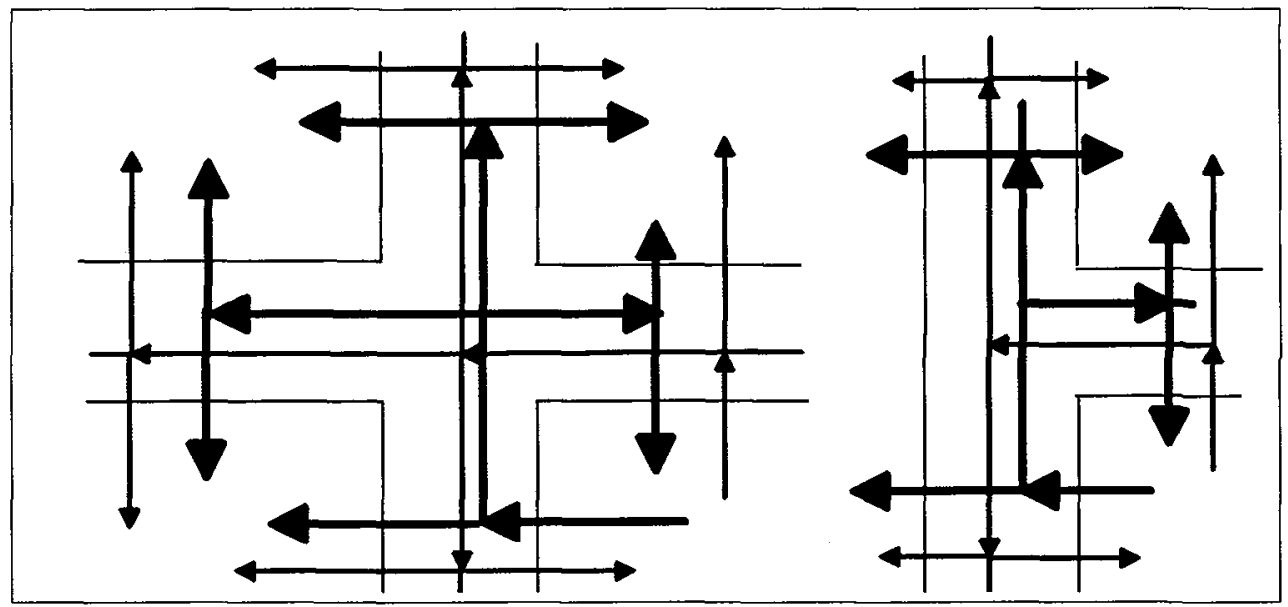

Fig. 1

Pour chacune des étapes de notre approche, nous avons appliqué la méthode de prévision des isolements acoustiques normalisés correspondante aux 150 cas de notre échantillon test.

Pour les jonctions en croix de parois liées rigidement, les différentes étapes ont conduit à la formule suivante:

$$
C_{i j}=0.15^{*}\left|R_{0}-R_{1}\right|+9
$$

Cette valeur est à comparer aux valeurs de $\left(D_{v, j i}+D_{v, j}\right) / 2$ données dans le document CEN/TC126/WG2 N61, soit

$$
8.7+5.7\left(\log m_{0} / m_{1}\right)^{2}
$$

Dans l'approche expérimentale du LASA, on retrouve un terme fonction du logarithme du rapport des masses des parois à travers la différence des indices d'affaiblissement acoustiques. Si on trace sur un même graphique les valeurs des facteurs de liaisons on obtient la figure 2 donnant les $L_{i j}$ en $d B(A)$ en fonction des rapports $m_{0} / m_{1}$. 
Les courbes B, C, D, E correspondent aux configurations suivantes:

Courbe $B$ : les deux parois sont lourdes $\left(m>150 \mathrm{~kg} / \mathrm{m}^{2}\right)$

Courbe D: Une paroi a une masse surfacique de $75 \mathrm{~kg} / \mathrm{m}^{2}$, l'autre est lourde

Courbe $E$ : Une paroi a une masse surfacique de $38 \mathrm{~kg} / \mathrm{m}^{2}$

Courbe $F$ : Les deux parois sont légères et rigides.

La courbe en pointillés correspond aux valeurs du document N61.

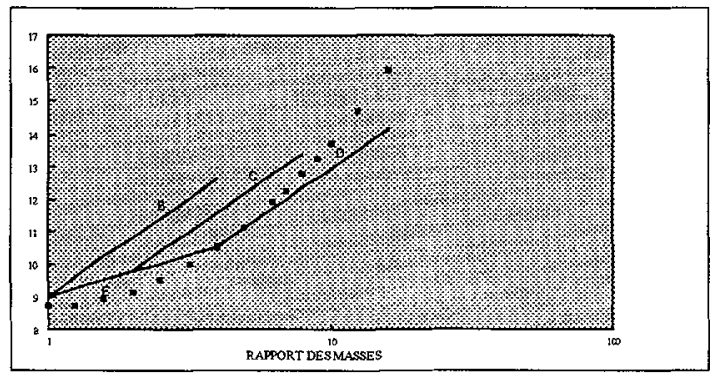

Fig. 2

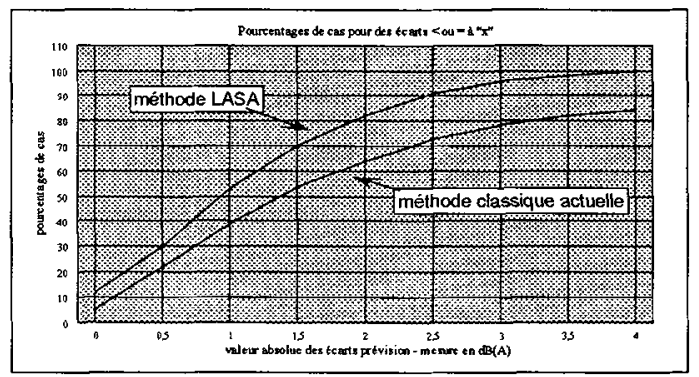

Fig. 3

On constate que pour les parois légères ou pour les liaisons entre une paroi légère et une paroi lourde les valeurs obtenues par l'approche expérimentale et celles du document N61 sont très voisines. Par contre la méthode du LASA conduit à des valeurs supérieures de 0,3 à $2 \mathrm{~dB}(\mathrm{~A})$ à la courbe du document CEN.

Nous avons tenté de modifier les valeurs LASA pour les rapprocher de la courbe CEN et de vérifier les écarts "prévisions - résultats de mesures" dans les cas de l'échantillon correspondants aux configurations en " $T$ " ou en croix relevant des courbes du document N61. Dans l'ensemble ces écarts avaient tendance à augmenter. C'est pourquoi nous sommes revenus aux valeurs issues de l'approche expérimentale qui avait pour principal mérite d'être sanctionnée par des résultats réels.

Rappelons que ces résultats réels correspondent à des opérations qui n'ont pas été particulièrement soignées sur le plan acoustique. Elles sont tout à fait représentatives des pratiques courantes de la construction. Notamment on ne connaît pas exactement la qualité des liaisons de parois à parois. II est vraisemblable que les liaisons entre parois lourdes de natures différentes ne sont pas aussi parfaites que celles réalisées en laboratoire ou que celles imaginées lors des approches théoriques. Des liaisons de mauvaise qualité, lorsque les parois latérales sont interrompues par la paroi de séparation ne peuvent être que favorables à la diminution des transmissions latérales, ce qui pourrait expliquer que les valeurs du LASA plus optimistes que celles de la théorie sont également plus proches des résultats réels.

Les courbes de la figure 3 donnent les pourcentages d'écarts "prévisions - mesures" constatés sur 108 cas (soit 424 liaisons) relevant des jonctions en croix ou en "T" avec des parois de séparation lourdes doublées ou non et des parois latérales non filantes. Les pourcentages sont ceux correspondant au nombre de cas pour lesquels l'écart prévision - mesure est supérieur ou égal, en valeur absolue, à x. On constate que la méthode développée par le.LASA permet de diminuer nettement l'imprécision due à la prévision.

La méthode LASA se présente sous la forme d'un programme informatique, baptisé MOD.L. Pour utiliser ce programme, qui fonctionne sous DOS, le constructeur n'a qu'à connaître les dimensions des locaux et la nature des parois les constituant. Le seul effort qui lui est demandé est de repérer sur un tableau de types de liaisons le cas correspondant à la jonction étudiée. II est ensuite très facile de modifier la nature de certaines parois, d'introduire des doublages, de modifier des dimensions afin d'optimiser les solutions permettant de viser une performance recherchée. 